Sílvia Lúcia Ferreira

Universidade Federal da Bahia

\title{
El movimiento feminista y la salud de las mujeres: la experiencia de los Centros de Planificación Familiar (CPF) en Catalunya $(1976-1982)^{*}$
}

Resumen: Este estudio pretende recordar las luchas del movimiento feminista de España (Catalunya) por el derecho a la salud de las mujeres analizando las estrategias utilizadas, que hicieron posible una mayor equidad de género en los Centros de Planificación Familiar (CPF), popularmente conocidos como plannings o planins. La información se recogió mediante entrevistas semi-estructuradas realizadas a mujeres, escogidas por su actuación en el movimiento feminista, y profesionales de salud que participaron en los centros mencionados en los municipios de Cerdanyola del Vallès, Barcelona y Santa Coloma de Gramenet. La creación y manutención de los plannings fue una victoria colectiva importante ya que unió estrechamente a muchas mujeres y produjo transformaciones sociales significativas.

Palabras claves: salud; movimiento feminista; planificación familiar.

Copyright (c) 2008 by Revista Estudos Feministas.

* Investigación realizada en el Programa de Postdoctorado, junto a la Universidad Autónoma de Barcelona, Depto. de Antropologia Cultural y Prehistoria, con Dra. Verena Stolcke como tutora. Apoyo: CAPES.

1 ÁVILA y CORRÊA, 2007.

\section{Introducción}

A pesar del reconocimiento casi unánime de investigadores que interpretan la emergencia del feminismo como un fenómeno del proceso de democratización, estamos de acuerdo con Betânia Ávila e Sonia Corrêa cuando escriben: "[...] casi nunca se afirma que la sociedad se democratizó, de hecho, porque las mujeres se organizaron y se convirtieron en protagonistas del dominio público [...]". ${ }^{1}$

Estas autoras afirman que la agenda construida y defendida por el movimiento feminista, en particular el sector de la salud, incidió directamente sobre elementos constitutivos de la lógica que simultáneamente disocia y articula lo público y lo privado: la maternidad como papel 
2 ÁVILA y CORRÊA, 2007.

${ }^{3}$ GORDON, 1980.

${ }^{4}$ GORDON, 1980. prioritario de las mujeres, la sexualidad, el papel de la profesión médica, así como la lógica de organización de los servicios públicos de salud. Esta agenda la fueron construyendo las mujeres mismas, a partir de la idea de reapropiación y resignificación de su cuerpo.

Deshacer el lugar del cuerpo (de la anatomía) como
destino, desconstruir la heteronomía en que estuvieron
(y aún continúan) inmersos los cuerpos femeninos son
los fundamentos de una concepción renovada de
ciudadanía, que incorpora las vivencias de la
sexualidad y de la reproducción y de esta forma abre
campo para que se supere el desposeimiento de sí,
experimentado por las mujeres en estas dos esferas.
Tal perspectiva exigía inevitablemente la elaboración
de nuevos derechos, sin los cuales la ciudadanía plena
de las mujeres no estaría satisfecha. ${ }^{2}$

La preocupación de las feministas por el tema de la sexualidad y la reproducción data de finales del siglo XIX. La historiadora Linda Gordon ${ }^{3}$ dice que el control de la natalidad es "una práctica humana tan genérica como la de cocinar o socializar a las criaturas". ${ }^{4}$ Para esta autora, a partir del desarrollo de la agricultura este control se fue disminuyendo con la pérdida del conocimiento y de las técnicas utilizadas por las mujeres, propiciando el recurso al aborto como un método para el control de la natalidad.

Gordon, a partir de la historia del feminismo americano, identifica tres fases de la ideología sexual en el feminismo: la primera, entre finales del siglo XIX y mediados del siglo XX, cuando el movimiento sufragista adquiere una ideología sexual que Gordon llama de "doméstica", con fuerte influencia del puritanismo sexual victoriano. La segunda, comprende el período cercano a la Primera Guerra Mundial, cuando los intereses de las mujeres se asocian con los movimientos de "liberación sexual", en los que también participan hombres, antecesores de los sexólogos modernos, como Havellock Ellis, Wilhelm Reich y Edward Carpenter. Marcan esta fase el apoyo a la actividad sexual fuera del matrimonio, la demanda de placer sexual y la crítica a la domesticidad. La última etapa, coincide con el surgimiento del feminismo actual a partir de finales de los años sesenta. En esta fase, se empieza a denunciar el papel que representa la familia en la represión sexual y en el control de la sexualidad de la mujer. Hay un cambio radical del significado de satisfacción sexual para las mujeres, una de las principales contribuciones históricas al movimiento de liberación de la mujer.

En el siglo XIX también había surgido un movimiento de maternidad voluntaria que reivindicaba el derecho a decidir sobre el número de hijos/as, lo que sirvió para 
${ }^{5}$ OLIVAN y GARAIZÁBAL, 1989.

${ }^{6}$ Pilar ESCARIO, Inés ALBERDI y Ana Inés LÓPEZ-ACCOTO, 1996, $\mathrm{p}$. 396. cuestionar la subordinación sexual específica de las mujeres y la relación entre hombres y mujeres. Este movimiento se constituyó como alternativa al movimiento conservador, que defendía la maternidad como el destino de las mujeres y razón básica para que éstas permaneciesen en el hogar. Mientras, las feministas afirmaban que la maternidad era la razón central para que las mujeres tuviesen mayor poder, independencia y respeto.

Para Olivan e Garaizábal, ${ }^{5}$ cuando se estudia la sexualidad de las mujeres en un determinado contexto, uno de los aspectos que se deben tener en cuenta es la tensión permanente que el placer y el peligro representan en la vida de las mujeres. A partir de esta tensión, dos grandes tendencias de elaboración teórica feminista dirigieron los estudios sobre el tema, apoyándose también en las raíces del siglo XIX. Una, insiste en el peligro que representa la sexualidad masculina para las mujeres, destacando su carácter violento y depredador; las mujeres se presentan exclusivamente como objeto de deseo, pocas veces como sujetos activos, y la sexualidad femenina es mucho más un impulso afectivo que un impulso erótico para el placer. La otra tendencia, más moderna y apoyada por el feminismo contemporáneo, insiste en el placer, en el deseo sexual de las mujeres, y se muestra contraria a dictar reglas y preceptos sobre lo correcto y lo incorrecto en sexualidad.

Esta variedad de tendencias sobre la sexualidad femenina acompaña a los discursos de las feministas a partir de la década de los 60, época del descubrimiento de la píldora anticonceptiva, que revolucionaría las costumbres y las prácticas sexuales de las mujeres de todo el mundo.

Este estudio tiene como objetivo la reconstrucción de una inusitada experiencia realizada por las mujeres y para las mujeres, recomponiendo, a partir del testimonio vivo, parte de la historia de los Centros de Planificación Familiar (CPF) en Catalunya. El período que se tomó para esta reconstrucción (1976-1982) demarca un cambio profundo en el que se hacen simultáneamente la transición política y la transición socio-cultural, y también es en este período cuando los plannings ideados por el movimiento feminista funcionan en toda su plenitud, hasta el I Congreso de Planificación Familiar realizado en noviembre de 1982 en Gijón, como un primer encuentro estatal de profesionales del sector:

[...] se llevan a cabo a la vez la implantación de las instituciones democráticas y las transformaciones culturales desde unos modos de vida homogeneizantes, tradicionales y conservadores a unas nuevas pautas de relación y de convivencia que se enmarcan en la diversidad y la tolerancia. ${ }^{6}$ 
7 SALGUERO, 2002, p. 55.

${ }^{8}$ En 1965, dos medidas gubernamentales suavizaron la postura oficial sobre la anticoncepción: una orden del Ministerio de la Gobernación permitía la distribución de anticonceptivos con prescripción médica, y otra, del Presidente del Gobierno, excluía de la categoría de delito el acto médico de recetar anovulatorios con fines terapéuticos.

La Universitat Autònoma de Barcelona (UAB), en 1974 y a través del Departamento de Ciencias Fisiológicas, ofreció un curso sobre Fundamentos de la Planificación Familiar. En ese mismo período también se registra una discusión sobre este tema en la Universitat de Barcelona (UB).

En 1976, un grupo de médicos de Barcelona firmó una carta en la que se pedía la revocación de la Ley de Protección de la Natalidad de 1941 y la solicitud para que se incluyera la planificación familiar en los programas de actuación sanitaria. La carta se presentó en el Colegio de Médicos. En los documentos analizados no hay ninguna referencia a la influencia del movimiento feminista en la realización de estos eventos. Es importante registrar la existencia de grupos feministas, con participación de asociaciones universitarias como la ANCHE (Associació de Comunicació Humana i Ecologia) creado en Barcelona en 1975 y la Asociación Universitaria para el estudio de los problemas de la mujer (AUPEM), entre otras.

${ }^{9}$ DEXEUS y PARERA, 1999

\section{El movimiento feminista en el período de transición democrática en España}

Durante todo el período de la dictadura franquista, hasta el 7 de octubre de 1978, los anticonceptivos estaban prohibidos. El Código Penal, en el artículo 416, declaraba que se podían castigar con penas de arresto mayor y multas de 5.000 a 100.000 pesetas a los que prescribieran, vendieran, divulgasen, ofreciesen, hiciesen publicidad o expusiesen públicamente objetos, instrumentos, aparatos, medios o procedimientos destinados a facilitar el aborto o a evitar la procreación.

Esta prohibición no alteró los niveles de fecundidad, que continuaban cayendo, independientemente de que el Estado aplicase, de forma deliberada, incentivos para que aumentase el número de hijos. A principios del siglo XX, el índice sintético de fecundidad en España estaba en 4,71 hijos por mujer, bajando paulatinamente hasta alcanzar, en 1950, los 2,46. En la segunda mitad del siglo hubo un rápido aumento, alcanzando 2,94 en 1965 y después una sucesiva caída, con la tasa más baja de Europa, con 1,16 en 1998 y 1,20 hijos por mujer en 1999. Este perfil de fecundidad se dio en momentos en que no existían métodos anticonceptivos eficaces. Cuando éstos se desarrollaron, las mujeres no tenían libre acceso a ellos por estar prohibidos. Magda T. Ruiz Salguero identifica que, a pesar de la prohibición y del rígido control por parte de la iglesia, cuando la píldora se comercializó en otros países,

muchas españolas trataban de conseguirla a través de amigas extranjeras o españolas que podían viajar a Francia o a Inglaterra. Lo mismo sucedía con el dispositivo intrauterino y el diafragma. La información sobre métodos más eficaces se fue generalizando a través de los grupos de mujeres y se fue creando una red de solidaridad en este sentido. ${ }^{7}$

Incluso antes de la transición política, iniciada después de la muerte de Franco en 1975, algunas iniciativas adoptadas por las corporaciones médicas, universidades y otras instituciones, precipitaron el fin de esta prohibición, facilitando y ampliando el acceso de más mujeres al uso de los contraceptivos. ${ }^{8}$ Dexeus y Parera ${ }^{9}$ reconocen que las consultas médicas privadas se liberaron rápidamente de las ataduras legales, favoreciendo el uso de contraceptivos hormonales a las mujeres con acceso a este tipo de asistencia, que correspondía a las que poseían mayor nivel de escolaridad, más liberales y progresistas, de clase mediaalta, con edad entre 20 y 30 años. Escario, Alberdi e LópezAccotto afirman que, en general, 
10 ESCARIO, ALBERDI y LÓPEZACCOTTO, 1996, p. 117.
11 Monserrat Cervera Rodon escribió "Com i on ens hem organizat les dones aquest 20 anys", en el libro 20 anys de feminisme, que incluye una memoria de los 20 años del feminismo catalán a partir de la primera jornada, realizada en 1976 (RODON, 1976).

12 ESCARIO, ALBERDI y LÓPEZACOTTO, 1996, p. 342. hay una primera etapa en que lo personal y lo político se presentan disociados, en el que las mujeres comienzan a verbalizar sus sentimientos, problemas y esperanzas a descubrir a través del diálogo, todo lo que hay de común en sus vivencias individuales, por una parte $\mathrm{y}$, por otra, a manifestar cierta incomodidad y disgusto con las maneras de hacer, pensar y vivir la militancia política que tienden a situarlas en el primer plano a la hora del trabajo y en el ultimo a la hora de la toma de decisiones. ${ }^{10}$

Esta fase, que mezcla lo personal y lo político, se desarrolla en los últimos años de la dictadura franquista. En 1975, año marcado por la prolongada enfermedad y consiguiente muerte de Franco, ya se conforman varias movilizaciones contra el régimen. En este contexto, los numerosos grupos de mujeres que se organizaron en el movimiento general de oposición al franquismo empiezan un camino propio, independiente.

La transición de la dictadura hacia la democracia es construida por las mujeres que llevan sus voces, sus sentimientos y sus propuestas al espacio público, en una diversidad de grupos y actividades. Independientemente del modo de actuación - más moderado o más radical ponen su pasión y su energía a disposición de un mundo diferente para hombres y mujeres.

Para Monserrat Cervera Rodon, ${ }^{11}$ los dos primeros años de los grupos y organizaciones están marcados por ideologías que se orientan por dos grandes corrientes de pensamiento: la corriente de la lucha de clases, con doble militancia y con orientación marxista, que defendía la emancipación de las mujeres a partir del socialismo y de la emancipación de la clase trabajadora. Estas mujeres eran militantes de partidos de izquierda, socialistas y comunistas y de grupos extraparlarmentarios. Las militantes eran casi todas jóvenes, algunas iniciándose en las profesiones liberales, muchas eran profesoras tanto de universidad como de primaria y secundaria, muchas trabajaban en fábricas o en servicios de salud y gran cantidad eran estudiantes.

En algunos casos estaban vinculadas a organizaciones o partidos en la lucha antifranquista, pero gran parte participaba en la oposición a la dictadura, sin vinculación a ningún grupo político constituido. La aproximación a las cuestiones políticas se daba por estos caminos o partiendo del trabajo de reflexión sobre los problemas específicos de las mujeres, incorporándose posteriormente a alguna organización político-partidaria. Escario, Alberdi e López-Acotto ${ }^{12}$ identifican la existencia de diversos colectivos feministas. 
13 ESCARIO, ALBERDI y LÓPEZACOTTO, 1996, p. 343.

\footnotetext{
${ }^{14}$ La convocatoria usada en este estudio fue divulgada por la Comisión Catalana de Organizaciones no Gubernamentales, compuesta por diversas entidades (acervo personal de una de las entrevistadas).
}

Esta multiplicidad de fóruns y entidades fragmentaba el movimiento feminista, que convivía con los conflictos generados alrededor de la doble o única militancia, si el feminismo debía inserirse en las luchas generales o constituirse como algo específico. También se le presentó otro dilema al movimiento feminista durante el período de transición política y, posteriormente, con la victoria de los socialistas: la ocupación o no de los espacios institucionales, cuando se cuestionaba si la lucha para cambiar las estructuras se debía realizar desde fuera o participar en ellas para transformarlas desde dentro. En el gobierno de transición de Suárez se desactivaron varias instituciones del franquismo, entre ellas la Sección Femenina, una de las instituciones que sustentó la política del gobierno dictatorial de Franco, como afirman las mismas autoras: "[...] había sido uno de los ejes de vertebración político-social de la dictadura, acorde a sus presupuestos doctrinarios de minoridad y sacrificio por parte de las mujeres, en aras de la grandeza de la familia y patria". ${ }^{13}$

La realización de las Jornades Catalanes de la Dona, en mayo de 1976, marcó la presencia de las mujeres catalanas organizadas que luchaban públicamente por sus derechos. Los términos de la convocatoria ${ }^{14}$ la definen como "un movimiento amplio y unitario donde las mujeres quieren participar activamente en la transformación de la sociedad hacia una humanidad libre e igualitaria en la que la mujer no sea utilizada como objeto de consumo, de placer, de reproducción biológica, de mano de obra barata y de perpetuación del sistema capitalista". La participación fue superior a 4.000 mujeres, con las siguientes delegaciones: 60 de Barcelona, 37 de otros puntos de Catalunya, 4 del País Valencià y 1 de Baleares.

Los puntos exhaustivamente debatidos en los días de las jornadas, realizadas en la Universitat de Barcelona (UB), trataban de la mujer y del trabajo, la legislación, educación, barrios, familia, medios de comunicación, sexualidad, política y la mujer y el campo. En estas jornadas se creó la Associació Catalana de la Dona y la Coordinadora de Vocalies i Grups de Dones.

Las reflexiones realizadas sobre el derecho de las mujeres a decidir sobre su propio cuerpo tuvieron en consideración la necesidad de anticonceptivos gratuitos, de una sexualidad libre y desvinculada de la procreación, del derecho al aborto atendido por la red pública de salud.

El grupo pionero en esta reflexión fue DAIA (Dones per l'Autoconeixement i l'Anticoncepció) que formulará una crítica concreta al sistema sanitario y, de modo especial, a la atención ginecológica. "Es época de desmitificar la virginidad, la maternidad, el pecado, de reivindicar el 
${ }^{15}$ RODON, 1996, p. 91

${ }^{16}$ a) Actitudes y percepciones de las mujeres respecto a su salud reproductiva y sexual, tesis de Doctorado de Mari Luz Esteban (ESTEBAN, 1993). Posteriormente publica una parte de la investigación: "La atención específica a las mujeres: 15 años de centros de planificación familiar" (ESTEBAN, 1994). La autora analiza la experiencia en el País Vasco.

b) La tesis de Doctorado de Iolanda Bodoque Puerta, realizada bajo la orientación de Mari Luz Esteban: Discursos y prácticas sobre sexualidad y reproducción: los centros de planificación familiar (PUERTA, 1996). La autora analiza una experiencia en Tarragona.

c) Un capítulo del libro publicado por el Instituto de la Mujer Lo personal es político. El movimiento feminista en la transición, con autoría de Pilar Escario, Inés Alberd y Ana Inés López-Acotto (ESCARIO, ALBERDI y LÓPEZ-ACOTTO, 1996).

\footnotetext{
${ }^{17}$ A efectos de este estudio y particularmente para la recolección de datos, a través de entrevistas, el término feminista tendrá un significado más amplio que el otorgado por la militancia; comprende a las mujeres que, organizadas o no en un grupo feminista, fueron más permeables a este discurso, y cuyas ideas y prácticas tenían como referencia directa o indirecta las teorías propugnadas por los colectivos feministas.
}

placer y desvincular la sexualidad de la reproducción, de denunciar el papel de la mujer reducida a una maternidad impuesta y a una fidelidad también impuesta." 15

A partir de estas primeras jornadas, se forman muchos grupos entorno a estas demandas y la idea central que empieza a desarrollarse es la creación de centros de información sobre anticoncepción y sexualidad, conocidos también como Centros de Planificación Familiar (CPF) o plannings.

Esta breve retrospectiva, que apuntaba algunos aspectos de esta compleja realidad vivida por el movimiento feminista catalán, nos da una idea de las dificultades que tenían que superarse para poner en práctica la idea de los CPF por las feministas.

A pesar de la importancia visible de los Centros de Planificación Familiar, sólo encontramos tres trabajos que traten de ellos de una forma un poco más detallada. ${ }^{16}$

Este es, por lo tanto, un relato colectivo construido a partir de narraciones de mujeres, con el que se intentan recomponer, a través de entrevistas y documentos, los acontecimientos y los hechos relevantes que sirvieron de base a la construcción y al funcionamiento de estos centros como espacios de actuación feminista. Es posible que hayan quedado fuera algunos acontecimientos, algunas posiciones divergentes o que algún aspecto pueda parecer incompleto, y que otras cosas hayan podido ser añadidas, matizadas por otras miradas. No pretendemos establecer una única versión de los hechos, se trata de recuperar un eslabón de la historia para que una vez más no se retroceda al espacio de la invisibilidad que caracterizó durante tanto tiempo la historia de las mujeres.

\section{Metodología}

La información básica para reconstruir la historia de los centros se recogió mediante entrevistas semiestructuradas, grabadas, realizadas por la investigadora a mujeres escogidas por su actuación en el movimiento feminista, y profesionales de salud que participaron en los centros mencionados y identificadas con el feminismo. ${ }^{17}$ Todas las entrevistadas atienden actualmente en centros de salud o en los sectores de anticoncepción, en los municipios de Cerdanyola del Vallès, Barcelona y Santa Coloma de Gramenet, fueron entrevistadas en el periodo de febrero a mayo de 2002 y autorizaron la divulgación de los resultados.

El análisis es realizado a partir de los testimonios de las entrevistadas, tomando como punto de partida la identificación de los centros, la formación de los equipos, y 
${ }^{18}$ El Dones per l'Anticoncepció i l'Avortament (DAIA) fue creado en 1976 y se constituyó como el grupo pionero y más activo en la lucha por el aborto libre y gratuito. cómo se daba la atención a las mujeres. Se retoma el proceso de transformación de los centros, apuntándose las principales rupturas del movimiento feminista en salud.

Para reconstruir la historia de los CPF en Catalunya en esta primera fase (1976-1982), entrevistamos a 7 mujeres que participaron directamente de esta experiencia:

Entrevistada 1: Enfermera. Participó desde 1982 en un Centre d'Atenció a la Dona i a la Parella en Mataró y después en un centro de atención y planificación familiar en Santa Coloma con orientación feminista.

Entrevistada 2: Doctora. Actualmente trabaja en atención primaria, con salud de la familia. Participó activamente en la creación del Centro de Planificación Familiar de Cerdanyola del Vallès. Su aproximación al movimiento feminista se dio a partir del cuarto año de Medicina, cuando pasó por la experiencia de un aborto y la posibilidad de trasladarse a otro país, ya que en España el aborto aún estaba prohibido.

Entrevistada 3: Actualmente es enfermera de un Centro de Atención Primaria en Cerdanyola y trabaja con jóvenes en programas de salud y sexualidad. Participó activamente en la creación del CPF de Ripollet.

Entrevistada 4: Médica de familia en la actualidad. Participó activamente en la creación del CPF de Ripollet como recién licenciada porque no aceptaba la forma como se atendían a las mujeres en los servicios de salud.

Entrevistada 5: Historiadora. Actualmente participa en el CAPS - Centro de Análisis y Programa Sanitario, una ONG de carácter interdisciplinario que promueve actividades científicas, de debate y reflexión sobre la salud. Fue una de las fundadoras de DAIA. ${ }^{18}$ Es militante del movimiento feminista desde principios de la década de los 1970.

Entrevistada 6: Doctora. Participó en la creación del primer Centro de Planificación Familiar de Barcelona y fue fundadora de DAIA; actualmente trabaja en el Centre Jove d'Anticoncepció i Sexualitat. Participa en el movimiento feminista desde las Primeres Jornades Catalanes de la Dona, aún como estudiante de medicina.

Entrevistada 7: Doctora. Participó en la creación de un Centro de Planificación Familiar y atiende actualmente un Centro de Atención Primaria. Fue una de las fundadoras de la Associació de Planificació Familiar de Catalunya i Balears. Participa en el movimiento feminista desde las Primeres Jornades Catalanes de la Dona, aún como estudiante de medicina. 


\section{Resultados y discusión: el funcionamiento de los plannings}

La idea de crear centros para atender a las mujeres desvinculados de la red de servicios de salud no era defendida por muchos de los políticos de izquierda ni por parte del movimiento sanitario. La postura de las mujeres se consideraba muchas veces radical, lo que convertía en más lento el proceso de creación de cada centro. También existía, en el mismo campo, toda la lucha por el aborto libre y gratuito, lo que posibilitaba posturas contradictorias: personas que apoyaban los centros, pero que públicamente no se comprometían con el derecho al aborto, o incluso conflictos en el interior del movimiento con relación al tema:

Yo fui de las iniciadoras de DAIA... bueno, fue un grupo muy intenso, yo creo, porque había mucha reflexión y había muchas ganas de discutir, de debatir, hacíamos debates, hacíamos muchas discusiones, había una buena relación, pero a la vez había posturas diferentes: el tema del deseo de la maternidad o no maternidad... Yo viví historias curiosas allí dentro, porque estuve embarazada de una de mis hijas y entonces había momentos en que no sabía si podía decir que estaba embarazada o no. Bueno, situaciones de esas que también eran mis situaciones, no sólo del grupo, esa especie de... dilema... de dilema contigo misma...

Los centros funcionaron desde el inicio como locales de debate y de formación feminista, además de atención ginecológica. Después del funcionamiento de los dos primeros, en El Prat de Llobregat (1977) y en Torre de Llobeta (1978), la lucha fue para conseguir un centro en cada pueblo, haciendo posible que un mayor número de mujeres tuviese acceso a la información y a los métodos anticonceptivos. En realidad, se trataba de construir espacios donde las mujeres pudiesen informarse sobre los aspectos recién descubiertos sobre la sexualidad femenina y de cómo estos podrían transformar la cotidianidad de sus vidas. También fue un entrenamiento para el uso de la voz en el espacio público, en una época en que las mujeres se expresan casi exclusivamente con amigas, familia y vecinos para resolver los aspectos de la vida cotidiana. Los espacios públicos eran masculinos y las voces públicas que se oían eran de los hombres. En este espacio de los plannings se valoraba que las mujeres se preparasen para decidir sobre su cuerpo, su sexualidad, si querían abortar o no, porque todo era completamente ilegal.

Era un poquito una lucha para eso, para poder hablar así en público como mujer y decir lo que estabas 
19 PUERTA 1996, p. 440.

pensando y reivindicar pues unos espacios separados, hasta que te aclararas. Yo creo que básicamente lo que queríamos era un sitio donde poder hablar, donde encontrarte con otras mujeres que también pudieras hablar, que nunca lo habías hecho, yo por lo menos no lo había hecho y bueno yo creo que era un poquito dejarnos oír, al margen de lo que opinaran nuestros maridos, compañeros y... podernos reunir como grupo de mujeres, que esto también era una cosa buena, mi compañero que era anarquista, otras que tenían sus maridos socialistas, en fin...

La abertura de un centro era una tarea muy difícil para las mujeres: las negociaciones se realizaban en varios grupos e instancias ya que se trataba de convencer a personas y de conseguir aliadas/os para defender un proyecto feminista revolucionario. El proceso democrático se estaba construyendo a pasos lentos pero las mujeres exigían espacios propios, con la construcción de una lógica inversa al camino burocrático seguido por los ayuntamientos. Esta lógica de negociación incluía la solidaridad y el apoyo de todas las que luchaban por los mismos objetivos y la resistencia física necesaria para dar cuenta de innumerables reuniones sin descuidar a los hijos, al compañero, a la familia y a los amigos.

Los grupos que se responsabilizaban de iniciar la discusión sobre la necesidad de los centros se formaban con mujeres que procedían de diferentes militancias, socialistas, comunistas, anarquistas, independientes en un proceso muy rico de discusión cuyas ideas rápidamente se difundían hacia otros lugares. Las negociaciones para que se abrieran los centros podían durar varios meses, y no siempre después de este plazo estaba asegurado el éxito del proyecto.

El estudio realizado por lolanda Bodoque Puertas ${ }^{19}$ sobre los centros de Tarragona identifica el esfuerzo que realizaron las mujeres para conseguir el funcionamiento de uno de los centros: después de meses de búsqueda de un local, finalmente consiguieron una casa que necesitaba reformas y tuvieron que pintarla y recoger algunos muebles usados, ya que el ayuntamiento no dio ninguna prioridad al proyecto. Este proceso no es diferente a los de otros espacios que se reivindican por y para las mujeres al poder público, al haber casi siempre una tradición de indiferencia con relación al mobiliario y a los materiales y equipamientos necesarios para su funcionamiento. Se cree que todos los espacios ocupados por mujeres son domésticos, semejantes a la casa y que por lo tanto "de puertas para dentro", todo puede funcionar sin necesidad de presupuesto. 
${ }^{20}$ Ayuntamientos con plannings: Barcelona (2); Cornellà de Llobregat; Barberà del Vallès; Esplugues de Llobregat; Gavà; L'Hospitalet de Llobregat (2); Manresa; Olessa de Monterrat; El Prat de Llobregat; Ripollet; Rubí; Sant Adrià del Besòs (2); Sant Andreu de la Barca; Sant Feliu de Llobregat; Sant Quirze de Vallés; Sant Vicenç del Horts; Sta. Coloma de Gramenet; Santa Perpètua de la Mogola; Terrassa; Viladecans; Tarragona; Valls (Tarragona); Badalona; Cerdanyola del Vallès; Molins de Rei; Montcada i Reixac; Sant Joan Despí; Sabadell.
${ }^{21}$ En la primavera de 1978 comenzaron los debates parlamentarios para revocar la ley de 1941. El Real Decreto sobre el establecimiento de Servicios de Orientación Familiar se aprobó en septiembre y autorizaba la apertura de 74 clínicas de planificación familiar patrocinadas por el Gobierno y distribuidas por todo el país, bajo la responsabilidad de la Subdirección General de Medicina Preventiva del Ministerio de Salud.

22 José LINHARD, 1984, p. 32.
Fueron identificados un total de 31 de estos centros ${ }^{20}$ distribuidos por Catalunya y que funcionaron a partir de 1978, ya que el año anterior sólo existía el del Prat de Llobregat.

Veinte uno de estos centros funcionaban con médicas, consultoras o monitoras y empleadas 0 administrativas. Seis de estos centros funcionaban con psicólogas o asistentes sociales y otros cuatro tenían equipos más completos, contando también con la presencia de comadronas. La denominación de consultora, monitora o planificadora fue una opción política encontrada para que no hubiese identificación de los centros con los servicios tradicionales y con los profesionales de la salud, caracterizando, de este modo, otro tipo de atención. La gran mayoría de las médicas que atendían en estos locales también era feminista o se identificaba con este discurso y tuvieron el valor suficiente para iniciar una atención que rompía con el modo tradicional realizado por los ginecólogos.

El camino para llegar a ser consultora pasaba por un proceso de participación en la militancia o incluso pasaba por la amistad, o por redes de información de personas permeables al discurso feminista y con las que se podía contar para desarrollar este tipo de trabajo.

\section{Transformarse para transformar: la formación de los primeros equipos}

A partir de 1976, concretamente después de las primeras Jornades Catalanes de la Dona, todos los esfuerzos se concentraron en la creación de los centros mencionados, que se constituirían en un patrimonio legado por el movimiento feminista: una alternativa de atención integral, revolucionaria, construida fuera del sistema sanitario, funcionando con doctoras y con algunas mujeres que no eran profesionales de salud, y con el control social que ejercía el movimiento de mujeres. ${ }^{21}$

En octubre de 1978 una enmienda del Código Penal autorizaba la venta, distribución y uso de anticonceptivos. La ley, aprobada en noviembre, amplió este derecho, dictando disposiciones para la concesión de licencias, fabricación, publicidad y venta de anticonceptivos. El aborto sólo se legalizó en 30/1 1/1983 con tres supuestos: problemas de salud para la mujer, problemas de salud para el feto y en caso de violación (estupro). ${ }^{22}$

En 1977 se abrió el primer centro en El Prat de Llobregat, y como los anticonceptivos aún estaban prohibidos, su prescripción se justificaba como medicina para las mujeres que tenían alteraciones. A partir de la 
${ }^{23}$ Funcionaba en el Po Maragall, 242.

${ }^{24}$ Los primeros centros funcionaron como laboratorio de autoaprendizaje para los equipos. La formación se daba a partir de diversas fuentes: el conocimiento técnico-científico a través de las médicas, la exploración y descubrimiento del propio cuerpo, las lecturas y los grupos de estudios realizados fuera de los horarios de funcionamiento de los centros y la elaboración del material didáctico en forma de cartillas que se distribuiría a las mujeres atendidas. ${ }^{25}$ Boston Women's Health Book Collective, 1976.

${ }^{26}$ Colectivo de Mujeres de Boston, 1976.

27 Leonor TABOADA. 1978.

${ }^{28}$ El self-help había empezado en 1971 en Los Angeles con Carol Downer y un grupo de mujeres que comenzaron a reunirse para desarrollar una técnica abortiva que pudiese liberar a las mujeres de la ley restrictiva de los EE.UU. En esta búsqueda comprendieron más profundamente que el control patriarcal de la vida reproductiva de una mujer moldea toda su existencia. De este modo, el self-help se transformó en un proceso de concienciación sobre la realidad de las mujeres, analizando también las estructuras capitalistas y patriarcales. Antes de un año, quedó clara la amenaza que representaban estas mujeres para el sistema médico convencional. Carol Downer fue apresada por práctica ilegal de la medicina. Pero en 1976 ya había clínicas en Alemania, Austria, Dinamarca, España, Estados Unidos, Francia, Holanda, Italia y Suiza. creación de este centro, los esfuerzos se concentraron en el sentido de construir un centro piloto en Barcelona, el Torre Llobeta, que empezó a funcionar a partir de $1978,{ }^{23}$ y cuya declaración de principios expresaba: tiene como objetivo la información a la persona en todos los aspectos relacionados con el conocimiento del cuerpo, desde un punto de vista anatómico y fisiológico, así como sobre las posibilidades de la mente para alcanzar la gratificación sexual.

Este centro se planeó en una coyuntura aún adversa, ya que las primeras elecciones democráticas sólo se realizaron al año siguiente y los anticonceptivos no se autorizaron hasta finales de año. ${ }^{24}$ Dos libros se constituirían como base para este aprendizaje: Our bodies, ourselves, ${ }^{25}$ traducido al español con el título Nuestros cuerpos, nuestras vidas; ${ }^{26}$ el segundo fue Cuaderno Feminista; introducción al self-help, escrito por Leonor Tabeada. ${ }^{27}$

\begin{abstract}
Entonces fuimos allí y nos pasaron unos folletos sobre temas de salud, supongo que nos informaron del libro este de las mujeres de Boston, que se convertiría en la Biblia, y bueno en la universidad también estaba Leonor Taboada que también fue... mi primer contacto... Fue a hacer un curso de cómo poner el espéculo en la universidad y... yo tenía entonces 18 años y me quedé asombrada... bueno primero que parecía una diosa griega... y empezó a hacer la técnica esta de "tú eres un espéculum en medio de la clase" y yo me quedé muy sorprendida ino? $Y$ esto quedó allí en el fondo.
\end{abstract}

Ambos libros se editaron con el mismo objetivo: promover la liberación de la mujer a través del autoconocimiento del cuerpo y de una vivencia plena de la sexualidad. Los grupos de autoayuda, autoconciencia o self-help tenían dos metas básicas: proveer educación sanitaria a todas las mujeres y ayudar a la mujer a auto realizarse. Los grupos de autoconciencia eran espacios de intercambio, de creación y de reflexión colectiva. El autoaprendizaje sobre el funcionamiento del propio cuerpo se desarrollaba en momentos de respeto y de complicidad. ${ }^{28}$

Es importante registrar que la divulgación de estas experiencias se hacía bajo forma de textos o libros que circulaban por correo o en congresos y que se traducían al idioma local. Otra forma de difusión era a través del desplazamiento de mujeres a países que desarrollaban estas experiencias y que posteriormente serían "multiplicadoras" en sus países de origen. Leonor Taboada enfatiza:

las mujeres que participaron en estos grupos revelaron sentimientos de fortalecimiento, de revalorización personal, confianza en si mismas y mayor capacidad para enfrentar sus problemas... Si una mujer plantea un encuentro entre médico y paciente y descubre 
29 TABOADA, 1978, p. 19.

30 TABOADA, 1978, p. 20. que otras mujeres tienen el mismo tipo de experiencias, cuestionarán como grupo las causas que han llevado a esta similitud y como esa interacción afecta a su posibilidad de conseguir un tratamiento. ${ }^{29}$

Los grupos no sólo alteraron la visión de la mujer sobre su cuerpo y su sexualidad, sino que promovieron un cambio social amplio a través del intercambio de informaciones forzando a las mujeres a una reflexión profunda sobre el marco social y a la construcción de un conocimiento colectivo con base a las propias experiencias. Como afirma Tabeada,

el self-help y el movimiento sanitario de las mujeres no son soluciones personales para problemas femeninos individuales, aunque éstas fueran, por sí solas, razones válidas para su existencia: son instrumentos para inducir al pensamiento y a la acción colectivos de las que pueda brotar un cambio social radical. ${ }^{30}$

Las mujeres que participaban en los grupos de autoconciencia se enfrentaban cotidianamente con situaciones de resistencia, tanto por parte de las amigas y amigos, como de familiares y, sobre todo, de los cónyuges, que no comprendían las sucesivas ausencias de casa para participar en reuniones cerradas. Las amenazas y las separaciones eran una constante en la vida de estas mujeres, porque intentaban introducir cambios en la vida cotidiana, principalmente en lo que se refería a la división del trabajo doméstico, a la vida sexual y a la creación de los hijos. En la cotidianidad de las familias se operaba una transformación casi silenciosa pero que provocaba incomodidad y celos, sobre todo por parte de los hombres, que veían cómo sus esposas, hermanas y compañeras trataban en público la sexualidad, hecho hasta entonces reservado al mundo masculino.

... lo hablábamos mucho, y a nosotras mismas nos fue muy bien participar... las trabajadoras... porque, bueno, hablábamos mucho de sexualidad en aquella época. Yo no he hablado nunca más tanto. Sí, porque fomentábamos mucho este debate, y los hombres, iban a decir... estaban recelosos de que nosotras estuviéramos tan activas y supiéramos tanto de sexo y había algunas que tenían problemas...De hecho, entre nosotras ha habido gente que ha tenido problemas. Gente que después se separaron el tal, y que los hombres dijeron que la separación estuvo en aquello.

Ésta también fue la época del destape y de la caída del puritanismo autoritario, en la que se da una exacerbación de comportamientos erótico-sensuales cada vez más explícitos por parte de los jóvenes y de parejas 
homo y heterosexuales y una vasta producción de libros y revistas dedicadas al sexo y a la sexualidad.

Los grupos de autoconciencia también se enfrentaban con las distintas reflexiones traídas por el movimiento feminista y que en cierta forma provocaban alguna diferencia en el modo de atender de cada planning.

La creación de los CPF consolidó a un grupo de mujeres que defendían un planning en cada pueblo, y caracterizó un movimiento por la planificación familiar en Catalunya. Los centros, a veces, dieron el apoyo necesario para que las mujeres que necesitasen abortar no tuvieran que desplazarse a otros países, práctica hasta entonces ampliamente realizada.

De esta forma, los equipos de trabajo se fueron formando a partir de las propias experiencias en un espacio de solidaridad e intercambio.

\section{¿Quiénes eran las mujeres que frecuentaban los plannings?}

No había un perfil determinado de mujeres que frecuentasen los centros, ya que dependía de los lugares en que estuviesen situados, generalmente estaban casadas y tenían hijos. Normalmente, los centros empezaban con una atención mínima que al poco tiempo se triplicaba, tanta era la necesidad de información que tenían las mujeres. Esta demanda elevada fue uno de los factores que aceleraron la transformación de los centros, puesto que no había voluntad política por parte de los ayuntamientos de desarrollar este proyecto. Las adolescentes frecuentaban poco este espacio porque no podían tratar del tema en presencia de sus madres o de mujeres más mayores.

Bueno, yo creo que no ha sido por el tipo de servicio sino por la evolución. Hay muchas jóvenes que tienen relaciones sexuales a edades más tempranas, que la permisividad es mayor... Entonces era una época muy oscurantista, sobre todo en el tema de las relaciones sexuales en jóvenes era diferente... y entonces no venían. En parte, también tuviera que ver el servicio, por la represión que había, como el centro estaba en el centro del pueblo y podían encontrarse tranquilamente a su madre en la sala de espera, entonces seguramente eso también influía, ¿no?

Las mujeres eran principalmente emigrantes de otras comunidades autónomas, como Galicia y Andalucía, y que ya habían tenido algún contacto anterior con el tema, ya que las feministas habían iniciado un trabajo intenso en los barrios sobre sexualidad, anticoncepción y aborto como parte de la estrategia de difusión de la ideología feminista. 
Este trabajo educativo realizado en los barrios y pueblos hizo posible que fuera madurando la viabilidad de los centros, creando expectativas y una demanda potencial de los mismos; por lo que no sorprende que en poco tiempo ya hubiese colas y listas de espera. Los primeros clientes eran casi siempre mujeres más próximas a los grupos feministas o incluso las que habían apoyado su apertura, ampliándose después a otras que eran bien atendidas, escuchadas y que solucionaban sus problemas de anticoncepción. Pero normalmente el centro de planificación se colapsaba al cabo de un año de haber abierto sus puertas.

Era un centro único en una población de 25.000 mujeres en edad fértil. Entonces yo pienso que los centros, bueno, esto es una crítica que se nos ha hecho... Ha sido un modelo pero que no ha respondido a las necesidades globales de la población, porque no daba más, porque es imposible por el equipo y por las prestaciones de un único centro para una población de 25.000 mujeres en edad fértil.

\section{La atención a las mujeres}

La crítica al modelo médico asistencial vigente trajo la posibilidad de crear una alternativa de atención diferenciada. Las mujeres que frecuentaban los CPF pasaban primero por una reunión (charla) en la que se les informaba sobre varios aspectos del funcionamiento de su cuerpo, que incluía anatomía y fisiología del aparato reproductor femenino, el ciclo hormonal y el papel de cada fase en la vida de la mujer, la sexualidad femenina y los condicionantes sociales que diferenciaban a las mujeres de los hombres, generalmente sumisas a ellos. También se discutía la libertad producida por los métodos anticonceptivos y el funcionamiento de cada uno. No se pretendía dar respuestas a las preguntas sino hacer que el grupo reflexionase sobre cada problema presentado. El tiempo dedicado a esta actividad variaba para cada centro pero casi siempre era superior a una hora porque se pretendía dar el máximo de información posible a las mujeres para que posteriormente pudiesen decidir sobre lo que hacer.

En un día normal las mujeres que estaban apuntadas por primera vez, en primera visita, lo primero que hacían era pasar primero por una charla, que duraba una hora y media, era larga. Entonces, era una charla donde se daban conocimientos pero fundamentalmente era un grupo de interacción en el cual las mujeres discutían y hablaban de lo que pensaban, el sea, que no era eso una charla en la que tú exponías 
y las demás escuchaban sino que pretendía ser una charla participativa. Y después de acabar la charla, entonces, ya se pasaba a la visita.

... Éramos bastante atrevidas, había también una parte de atrevimiento, de juventud, de tener unas ideas $y$ aunque a lo mejor no estábamos muy entrenadas en lo profesional, sí que teníamos unas idea claras y estas ideas eran innovadoras, $y$ creo que esto fue importante. La formación vino... posterior. Primero empezamos a preguntarles a las mujeres sobre su sexualidad $y$ después nos preguntamos qué les teníamos que decir nosotras, y aquí fue cuando nos formamos profesionalmente, pero primero tuvimos el atrevimiento de preguntarlo. $Y$ de preguntarlo en un país donde esto nunca se había tratado, no se había hecho... y esto yo creo que fue una cosa importante, y no hablo como un acto individual, sino como un acto colectivo...

Después de estas charlas se realizaba una entrevista personal y una consulta ginecológica. Algunas veces se incorporaba la entrevista personal a la consulta médica. La ficha era un impreso dactilografiado que incluía una investigación detallada sobre la vida de las mujeres. Además de los aspectos relacionados con los antecedentes familiares, se les hacía varias preguntas relacionadas con su vida sexual y afectiva: las preferencias sexuales (homosexualidad), hábitos de masturbación, presencia o no de orgasmos y cómo se conseguían, el valor de la relación sexual en la vida cotidiana, tipo de eyaculación del cónyuge/ compañero (normal, rápida o precoz). Las prácticas anticonceptivas también se investigaban detalladamente: cómo se sentía con cada método usado, la tolerancia orgánica, los fallos, las motivaciones para usar determinados métodos y las alteraciones y adaptaciones que el método comportaba en la vida cotidiana.

La atención realizada por la médica rompía con el tradicional modo de atender usado por la ginecología hasta el momento, puesto que la historia clínica incorporaba varios aspectos de la sexualidad femenina y estas informaciones se tomaban en cuenta en el momento de establecer las conductas terapéuticas. El diálogo sobre un aspecto hasta entonces íntimo y personal, como la vida sexual, aproximaba a estas mujeres, y el conocimiento sobre su cuerpo, socializado y rediscutido, era el punto de unión entre estos dos mundos femeninos. La atención de las médicas también incluía el aprendizaje de la autoexploración del cuerpo, como el examen de las mamas y la visualización de la vagina y el cuello del útero, mediante el uso de un espejo. Se estimulaba a todas las mujeres atendidas a tocarse y a autoconocerse para distinguir los cambios corporales en 
las diversas fases de la vida. La prescripción de un método anticonceptivo escogido requería siempre mucho cuidado, principalmente en lo que se refiere a las píldoras anticonceptivas, que en aquella época ofrecían muchos más riesgos. En la consulta ginecológica también se hacía la prescripción de medicinas para los problemas ginecológicos presentados.

Organizamos una historia clínica en la que se recogieran más o menos todas las cosas que nosotros queríamos y pensábamos que eran importantes de recoger respecto a la salud de las mujeres. Es la primera vez que introdujimos el tema de la sexualidad en una historia clínica, y esto es importante porque esto lo pensamos desde la individualidad de cada una pero también desde un colectivo de mujeres que estábamos más el menos todas, bueno claro, yo estaba en Sant Andreu de la Barca, pero otras estaban en otros puntos. Después, primero cada uno hizo un poco lo que le parecía y después esto lo llegamos a unificar, prácticamente hubo un tipo de... un modelo de historia clínica que fue lo que nos parecía más interesante, que recogía mejor las cosas que nos parecían importantes en este tema.

Después de la charla y entrevista/consulta, las mujeres tenían mayor claridad sobre el método más adecuado a su situación y que les podría dar mayor libertad. Gran parte de estas elecciones recaía sobre la píldora o el DIU. El uso del diafragma se fue introduciendo poco a poco, ya que el equipo necesitaba más tiempo para identificar, con el propio uso, los beneficios y las dificultades.

Algunas decían: iuy no!, pero entrábamos en el tema y les decíamos: "te tienes que conocer y tocarte, no pasa nada, y la vagina"... Incluso habíamos hecho con el diafragma, juntas, talleres para aprenderlo a poner, y una lo ponía a la otra, e incluso con el espejo... Sí, eso en el curso, nosotras nos lo hicimos todas.

Con el paso del tiempo y de más conocimiento, en las charlas se puso cada vez más énfasis en el diafragma, porque su uso posibilitaba un mayor conocimiento sobre el cuerpo.

${ }^{31}$ ESTEBAN, 1994, p. 21.

Mari Luz Esteban, ${ }^{31}$ a partir de la evaluación realizada por las propias mujeres acerca de los CPF, afirma que éstas valoraron la atención cuando se comparaba con otras consultas ginecológicas, tanto de la red pública como de la privada. Entre las características que las diferencian están: a) el tipo de información ofrecida, dando valor a los aspectos más cualitativos; b) el tiempo dedicado a las informaciones y la tendencia a hacer uso de actividades grupales; c) el abordaje directo y diferente de cuestiones relativas a la vida 
32 ESCARIO, ALBERDI y LÓPEZACOTTO, 1996, p. 229.

${ }^{33}$ La Coordinadora Estatal de los Grupos de Plannings familiar es una instancia unitaria que agrupa a todos los grupos feministas a escala nacional. En abril de 1977 celebran una asamblea estatal a la que asisten representantes de Gerona, Lérida, Euzkadi, Madrid y Barcelona. sexual y a la situación personal y social de las mujeres; d) la resolución inmediata del motivo que provocaba la visita, un embarazo no deseado, por ejemplo; e) el contacto realizado por los CPF con otras entidades, como centros escolares, asociaciones de vecinos etc. Otros aspectos subjetivos se valoraron como positivos: sentirse bien tratada y ser escuchada, entre otros.

Uno de los problemas encontrados por los plannings desde su aparición fue la distribución gratuita de anticonceptivos, una de las reivindicaciones del movimiento feminista. Muchos ayuntamientos no disponían de infraestructura administrativa para este tipo de demanda, además de una ausencia clara de voluntad política ampliamente expresada por las entrevistadas. Muchas mujeres con problemas ginecológicos o que tomaban píldoras necesitaban exámenes complementarios, lo que tampoco aseguraba la red pública.

De esta forma, los plannings convivían con el aumento progresivo de la clientela y con varios problemas estructurales que demandaban resolución inmediata del poder público. Parte de estos problemas se resolvía por la red de voluntariado que se formaba alrededor de los centros.

\section{Los primeros cambios}

La Jornada de Granada, realizada en 1979, marca el inicio de una profunda crisis en el movimiento feminista.

Las formas de plantearse los debates en las jornadas convocadas por la Coordinadora Estatal provocaran el abandono de muchas mujeres - hasta mil, según textos de la época, cansadas de la capitalización por los partidos, la rigidez de la organización y el poco interés de las ponencias. En sesiones paralelas se trataban de temas puramente feministas que fueron seguidos por gran interés, durante todas las jornadas. ${ }^{32}$

Los debates en torno a la doble militancia y la militancia única fueron ásperos y, debido al clima emocional generado, no se llegó a ningún acuerdo. Estas jornadas marcaron una división fundamental, ya que el punto de mayor tensión estaba entre las que asociaban la lucha de las mujeres a los partidos políticos y las que pretendían un camino independiente de los partidos. En estas jornadas, no hubo conclusiones colectivas ni plataformas conjuntas de acción.

El período de 1979 a 1982 en Catalunya también está marcado por una transformación de los grupos. De manera coordinada, continúa funcionando la Coordinadora de Vocalies de Barris y la de los centros de planning. ${ }^{33}$ En 1982 el Partido Socialista Obrero Español (PSOE) gana las 
${ }^{34}$ RODON, 1996, p. 26.

${ }^{35}$ Para entender el debate en torno a la creación del Instituto de la Mujer ver ESCARIO, ALBERDI y LÓPEZ- ACOTTO, 1996, p. 288294. elecciones estatales y genera una movilización y la esperanza de que se cumplan todas las promesas que habían hecho durante la campaña. Muchas feministas van a trabajar a los ayuntamientos de izquierda haciendo un trabajo feminista a partir de las instituciones. ${ }^{34}$ Con el gobierno socialista muchas de las transformaciones se fueron implementando, como la creación del Instituto de la Mujer (1983), resultado de las presiones de un grupo de mujeres del Partido Socialista que adoptaron como modelo las experiencias de los países nórdicos, de Francia y Alemania, los cuales, en años anteriores y con gobiernos socialistas, habían creado instituciones de defensa de los derechos de las mujeres. ${ }^{35}$ En el conjunto de estas transformaciones se incluía la estructuración de los servicios de salud, con grandes cuestiones por resolver ya que la interrupción voluntaria del embarazo (aborto) y la esterilización voluntaria continuaban siendo ilegales.

Hasta 1982, por lo tanto, en lo que se refiere a la asistencia a la salud de las mujeres, funcionaban los CPF de orientación feminista, una red de atención primaria y también las clínicas privadas.

En el I Congreso nos encontramos con profesionales de diversos orígenes. Unos provenientes de la medicina tradicional, $y$ otros provenientes de los centros municipales, que entendían que la Planificación Familiar tenía un componente más de educación sanitaria que lo de asistencia médica.

Este Primer Congreso de Planificación Familiar tuvo lugar en Gijón (Asturias), en los días 19 y 20 de noviembre de 1982, bajo la responsabilidad de la comisión de salud del ayuntamiento y pretendió reunir por primera vez a los distintos colectivos profesionales dedicados al tema para identificar la problemática existente para una posterior integración.

El congreso de Gijón fue un punto de salida para muchas cosas. Llevábamos un año reuniéndonos, en este año conseguimos elaborar esta ponencia con diez puntos que eran un poco la filosofía que teníamos para la planificación familiar, lo que nosotros queríamos que fuera la planificación familiar en España. Y a partir de aquí decidimos ya trabajar para constituirnos en una asociación aquí en Catalunya, pero también trabajar para constituir una asociación a escala de todo el estado. Esto fue como el punto de partida de todo lo que después ha sido el movimiento de planificación familiar ya más organizado.

En los documentos del congreso se registra la protesta por parte de las consultoras de que algunas discusiones de los grupos habían degenerado en problemas entre ciertos 
${ }^{36}$ La Comisión Gestora Nacional de Planificación Familiar estuvo formada por Mercè Gascó, Rosa Ros y Encarna Albella (Catalunya), Isabel Serrano, Rita Daudén y Marisa Castro (Madrid), Federico Goyarts y Carmen Olmedo (Andalucía), Amparo Cardaño y Luis Monasterio (Euzkadi), Pilar Alonso (Asturias), Leonor Taboada (Baleares), Antonio Campos Romay (Galicia) e Isabel Soler.

37 El $2^{\circ}$. Congresso de Planificación familiar se realizó en $\mathrm{A}$ Coruña, en marzo de 1985. profesionales y que, en principio, sólo esperaban discutir los criterios de atención y las formas de integración entre los diversos colectivos profesionales.

Hay, en este congreso, por lo tanto, una serie de rupturas que necesitan ser puntuadas: la preocupación por parte de las feministas de presentar, en este momento, los puntos imprescindibles sobre lo que desean de una planificación familiar, reafirmando su compromiso con las mujeres. Esta plataforma de 10 puntos fue discutida en reuniones realizadas en el año anterior y contenía los principios generales sobre la asistencia a las mujeres. La idea de una red de Planificación Familiar también se apuntó, pero posteriormente se rechazó porque se contraponía a una red única de servicios de salud.

Los profesionales de la salud ya pretendían conseguir un espacio diferenciado, y los documentos apuntaban a la necesidad de discutir la sexualidad con personas con formación específica: psicólogos y sexólogos.

Esto es lo que se inicia en el congreso de Gijón, aquí es donde se inicia el recorrido; el movimiento de planificación familiar como una entidad separada del movimiento feminista, pero no al margen ni en contradicción o contraposición, no. Simplemente diferente. Es algo diferente, si no, no se hubiese generado una asociación de planificación familiar, una federación de planificación familiar, porque tiene una entidad propia que es distinta del movimiento feminista.

En este congreso también se aprobó la creación de una Comisión Gestora Estatal de Planificación Familiar, ${ }^{36}$ que tenía los siguientes objetivos: difundir las conclusiones del congreso, coordinar a los profesionales del sector y preparar el segundo congreso. ${ }^{37}$

En el período de 1982 hasta marzo de 1984 se realizaron jornadas en las que se discutieron las conclusiones del congreso y las nuevas bases de un modelo asistencial que integraría a los plannings en una red de atención primaria.

Los plannings fueron siendo paulatinamente descaracterizados en su proyecto original: se transfirieron las médicas y consultoras a Centros de Atención Primaria (CAP) $y$, en algunos casos, recibieron profesionales de la salud que cambiaron toda la dinámica de trabajo. Se cerraron unos cuantos después de un período de funcionamiento de aproximadamente dos años por la imposibilidad de continuar la atención por exceso de clientela y principalmente por falta de recursos por parte de los ayuntamientos. También hubo conflictos generados por profesionales de la salud con respecto a la formación de las consultoras y de la ausencia de título de especialista en 
Ginecología por parte de las doctoras que atendían en los centros. Los centros restantes, que esquivaron estos conflictos, fueron siendo gradualmente transformados en Centres d'Atenció Primària (CAP) o Centros de Atención a la Mujer y actualmente integrados en la red sanitaria.

\section{Conclusiones}

La parte del movimiento feminista que se volcó de modo más directo en las cuestiones de salud y sexualidad defendía la anticoncepción, el derecho al aborto libre y gratuito y la amplia información para las mujeres, además de un cambio radical en la forma en que los servicios de salud atendían a las mujeres. En algunos momentos estas reivindicaciones se hicieron sobre un solo eje y, en otros, se dividió, cuando la lucha por el aborto continuaba pero de una forma más independiente, porque que los anticonceptivos ya estaban permitidos. En ese momento también resultaba imprescindible una estructura de atención que diese un poco de seguridad a las mujeres que deseaban realizar el aborto en condiciones seguras sin tener que desplazarse a otros países, práctica hasta entonces ampliamente realizada.

Esta separación por veces se interpreta como un movimiento distinto, en el que los plannings se habían creado mucho más para responder a una necesidad de todas las mujeres que aspiraban a tener a su disposición métodos anticonceptivos modernos y ya no se sometían a prohibiciones. Esta aspiración fue impulsada por el movimiento feminista que transformó el deseo en acción.

Algunas entrevistadas reconocen que el movimiento feminista perdió el espacio conquistado, dejó que éste se le escapase de las manos sin muchas protestas, y esto demuestra un retroceso de las conquistas de las mujeres, ya que se había obtenido a costa de mucho sudor y lucha. A pesar de ello, reconocen que fueron muchas las ganancias que obtuvieron el propio movimiento, las mujeres y la reorganización de los servicios de salud.

Las entrevistadas creen que el uso del coitus interruptus por parte de la población, al contrario que la participación y la corresponsabilidad masculina, demuestra precisamente un mayor control de los hombres sobre las mujeres y la ausencia de negociación entre la pareja. En los plannings, se incentivaba poco este método porque no permitía que la mujer dijera no al compañero. Como él tenía la responsabilidad del control, no veía motivos para una abstinencia sexual, muchas veces necesaria para las mujeres. Con este método muchas mujeres contaban que casi nunca sentían placer por el miedo de quedarse 
embarazadas o porque los tiempos eran diferentes, lo que las dejaba insatisfechas.

También había una cierta tradición en el uso de este método que pasaba de padres a hijos, incluso en la ausencia de diálogo entre ellos.

La creación y manutención de los plannings fue una victoria colectiva importante ya que unió estrechamente a muchas mujeres y produjo transformaciones sociales significativas. El hecho de que estos espacios se transformasen en una red oficial de prestación de servicios, en algunos casos ajenos o conflictivos con el movimiento feminista, no anula o disminuye su importancia en un determinado período y contexto.

\section{Referencias bibliográficas}

ÁVILA, Maria Betânia; CORRÊA, Sonia. O movimento de saúde e direitos reprodutivos no Brasil: revisitando percursos. Católicas por el Derecho a Decidir. Disponível em: http:/ /www.geocities.com/catolicas/articulos/art13.html. Acesso em: set. 2007.

BOSTON WOMEN'S HEALTH BOOK COLLECTIVE. Our bodies, ourselves. New York, 1976.

COLETIVO DE MUJERES DE BOSTON. Nuestros cuerpos, nuestras vidas. Barcelona: Ed. Fontanella, 1976

DEXEUS, S.; PARERA, N. Perfil de la usuaria de los métodos contraceptivos. Memoria del XII Simposio de la Sociedad Catalana de Anticoncepción. Barcelona, marzo 1999.

ESCARIO, Pilar; ALBERDI, Inés; LÓPEZ-ACOTTO, Ana Inés. LO personal es político. El movimiento feminista en la transición. Madrid: Instituto de la Mujer. 1996.

ESTEBAN, Mari Luz. Actitudes y percepciones de las mujeres respecto a su salud reproductiva y sexual. Tesis de Doctorado. Universitat de Barcelona, 1993.

. "La atención especifica a las mujeres: 15 años de Centros de Planificación Familiar". Cuadernos de Salud Pública, n. 15, 1994.

- Re-producción del cuerpo femenino. Discursos y practicas acerca de la salud. Bilbao: Tercera PrentsaHirugarren Prentsa, 2001.

GORDON, Linda. "La lucha por la libertad reproductiva: tres etapas del feminismo". In: EISENSTEIN, Z. (Comp.). Patriarcado capitalista y feminismo socialista. México: Siglo XXI, 1980. p. 124-149.

LINHARD, José. "La planificación familiar en España". Perspectivas Internacionales en Planificación Familiar, número Especial, p. 30-36, 1984. 
OLIVAN, M.; GARAIZÁBAL, C. "Introducción". In: VANCE, Carole S. (Comp.). Placer y peligro, explorando la sexualidad femenina. 2. ed. Madrid: Talasa Ediciones, 1989. p. 5-8.

PUERTA, lolanda Bodoque. Discursos y prácticas sobre sexualidad y reproducción: los centros de planificación familiar. Tesis de Doctorado. Universitat Rovira i Virgili, Tarragona, 1996.

RODON, Montserrat Cervera. "Com i on ens hem organitzat les dones aquest 20 anys". In: 20 anys de feminisme. 1996. p. 22-29.

RODRIGUES, Manola; MARGALEF, Montse; BOFILL, Mireia; CASALS, Nuria. "Presentació". In: 20 anys de feminisme. 1996. p. 8-15.

SALGUERO, Magda Tereza Ruiz. La regulación de la fecundidad: un estudio demográfico de la anticoncepción, la esterilización el aborto y el tratamiento de la esterilidad en España. Tesis Doctoral. Departamento de Geografía, Centro de Estudios Demográficos, Programa de Doctorado en Geografía, Universitat Autònoma de Barcelona, 2002.

TABOADA, Leonor. Cuaderno Feminista. Introducción al self help. Barcelona: Editorial Fontanella, 1978. Edición agotada y no-reeditada.

[Recebido em setembro de 2007 e aceito para publicação em fevereiro de 2008]

\section{The Feminist Movement and Women's Health: The Experience of the Family Planning Centers (CPF) in Catalunya (1976-1982)}

Abstract: This study seeks to remember the fights of the feminist movement of Spain (Catalunya) for the women's right to health care, through the analysis of the strategies that made possible a better gender equity in the Centers of Family Planning (CPF), popularly known as planning or planins. The basic information to reconstruct the history of the centers was picked up by semistructured interview carried out with women, chosen by their performance in the feminist movement, and health professionals who participated in the mentioned centers in the municipalities of Cerdanyola of the Vallès, Barcelona and Santa Coloma of Gramenet. The creation and maintenance of the planning was an important collective victory since it united many women closely and it produced significant social transformations.

Key Words: Health; Feminist Movement; Familiar Planning. 